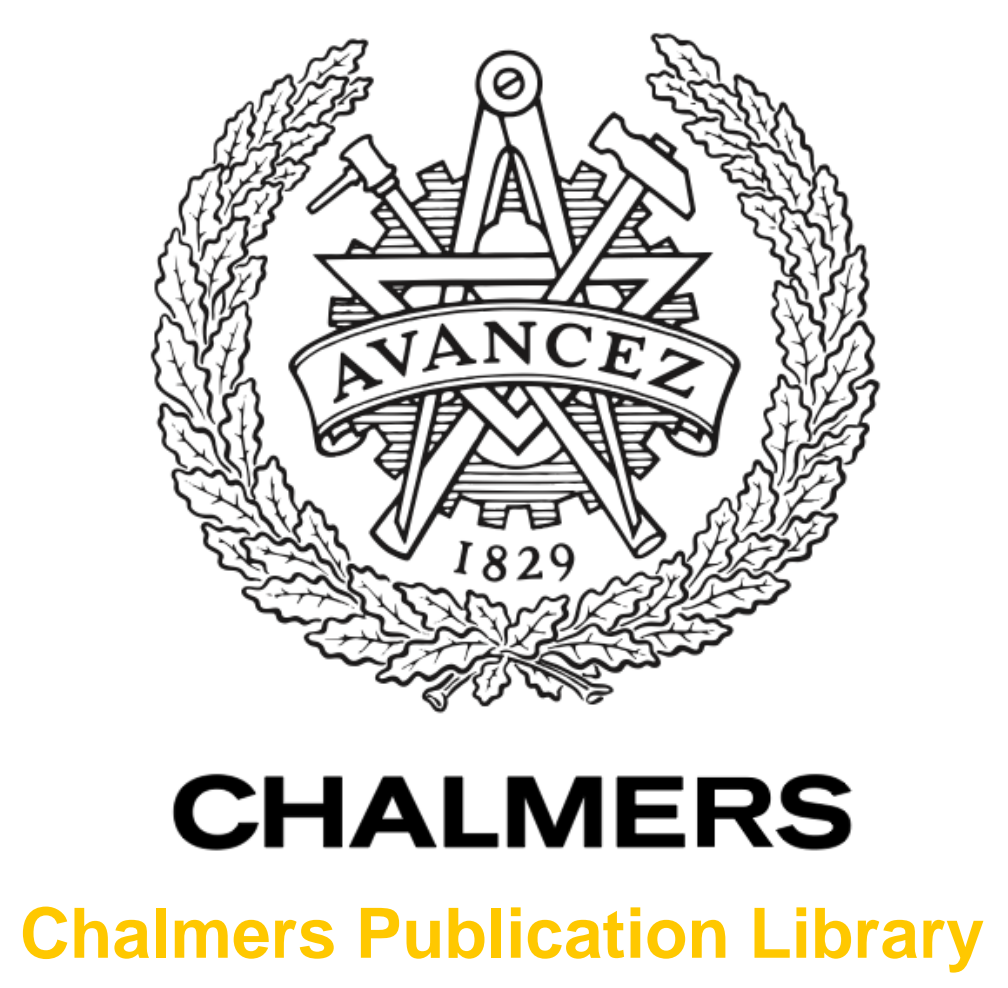

\author{
Intersymbol interference penalties for OOK and 4-PAM in short-range optical \\ communications
}

This document has been downloaded from Chalmers Publication Library $(\mathrm{CPL})$. It is the author's version of a work that was accepted for publication in:

\author{
2013 Optical Fiber Communication Conference and Exposition and the National Fiber Optic \\ Engineers Conference, OFC/NFOEC 2013 \\ Citation for the published paper: \\ Szczerba, K. ; Karlsson, M. ; Andrekson, P. et al. (2013) "Intersymbol interference penalties \\ for OOK and 4-PAM in short-range optical communications". 2013 Optical Fiber \\ Communication Conference and Exposition and the National Fiber Optic Engineers \\ Conference, OFC/NFOEC 2013 \\ http://dx.doi.org/10.1364/OFC.2013.OW4A.3
}

Downloaded from: http://publications.lib.chalmers.se/publication/186986

Notice: Changes introduced as a result of publishing processes such as copy-editing and formatting may not be reflected in this document. For a definitive version of this work, please refer to the published source. Please note that access to the published version might require a subscription. 


\title{
Intersymbol Interference Penalties for OOK and 4-PAM in Short-range Optical Communications
}

\author{
Krzysztof Szczerba, Magnus Karlsson, Peter A. Andrekson, Anders Larsson. \\ Photonics Laboratory, Department of Microtechnology and Nanoscience, Chalmers University of Technology, \\ 412-96 Gothenburg, Sweden. \\ krzysztof.szczerba@chalmers.se
}

\begin{abstract}
Intersymbol interference penalties of OOK and 4-PAM were compared in the context of short-range optical links with directly modulated VCSELs. The maximum achieved bit rate with 4-PAM was 38 Gbps and 32 Gbps with OOK.
\end{abstract}

(C) 2012 Optical Society of America

OCIS codes: (060.2330) Fiber optics communications; (060.4080) Modulation.

\section{Introduction}

Growing demand for data throughput in short-range optical communications has motivated investigation of multilevel modulation formats. Such communications systems predominantly use directly modulated, high speed and low cost vertical cavity surface emitting lasers (VCSELs), multimode fiber (MMF) and direct detection. Many modulation formats have been investigated, for example various forms of single and multiple subcarrier modulation [1,2], pulse amplitude modulation (PAM) [3] as well as many other formats. Short-range optical communications in a datacenter is subject to very stringent constraints on power consumption and cost, which limit the practical complexity of the modulation used. In such an environment, simple multilevel formats, such as PAM are most likely to be applied.

Multilevel modulation formats with higher spectral efficiency could increase the bit rate in a given bandwidth, but on the other hand require more optical power to reach sufficiently low bit error rate (BER). On the other hand, if the symbol rate of OOK increases, for a given link, so does the intersymbol interference (ISI) penalty. From the sensitivity point of view, the multilevel modulation becomes better than OOK at the bit rate for which the ISI penalty of OOK would increase over the additional power required for multilevel modulation.

Results of an investigation of the ISI penalties of 4-PAM and OOK with fixed bit and symbol rates and varying fiber lengths were presented in [4]. In this work we investigate the effects of the ISI penalties when the frequency response is fixed (with $-3 \mathrm{~dB}$ bandwidth equal to $8.5 \mathrm{GHz}$ ), and the bit rate is increased. Maximum achieved bit rates were 38 Gbps with 4-PAM and 32 Gbps with OOK. All BERs were measured in real time down to $10^{-12}$.

\section{Theoretical sensitivity and ISI}

The theoretical BER of an M-PAM modulation, under assumption that the system is dominated by thermal noise, modulation levels are equally spaced, and Gray labeling is used, is given by:

$$
B E R=\frac{M-1}{\log _{2}(M) M} \operatorname{erfc}\left(\frac{I_{\text {avg }}}{(M-1) \sqrt{2} \sigma}\right),
$$

where $I_{\text {avg }}$ is the average photodetector current and $\sigma$ is the root mean square noise current [4]. If natural labeling is used instead of Gray labeling, the BER is slightly higher in the high BER regime, but it is well approximated by Eq. 1 in the low BER regime [4]. The theoretical power penalty for using M-PAM instead of OOK at the same bit rate is $P_{p b}=10 \log _{10}\left((M-1) /\left(\sqrt{\log _{2}(M)}\right)\right)$, which in the case of 4-PAM means $3.3 \mathrm{~dB}$ more optical power required to reach the same BER as OOK [4]. This does not include the ISI penalty for OOK, however. The theoretical ISI penalty can be can be calculated from the eye closure by

$$
P_{I S I}=10 \log _{10}\left(\frac{1}{1-E_{m}}\right)
$$




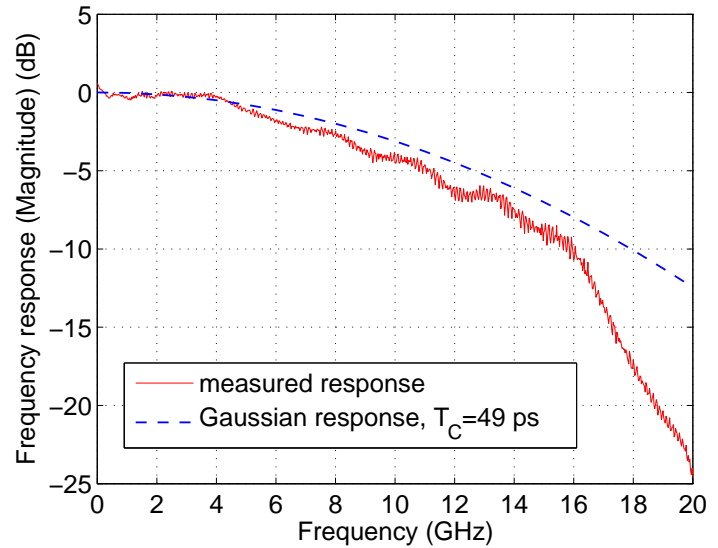

(a)

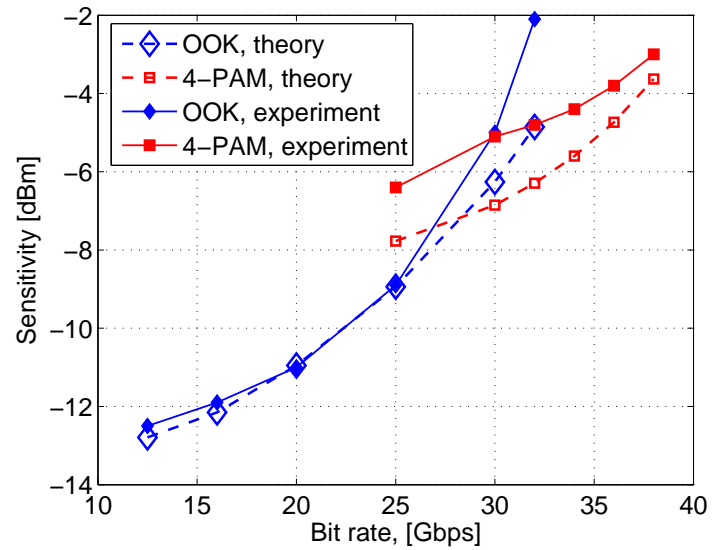

(b)

Fig. 1: Magnitude of the frequency response of the tested system, together with Gaussian response corresponding to $49 \mathrm{ps}$ rise time (a) and the theoretical and experimental sensitivity including the ISI penalty for 4-PAM and OOK (b).

where $E_{m}$ is the eye closure. Assuming that Gaussian pulses are used, and that the impulse response of the system has Gaussian shape, the worst case eye closure for OOK is approximated by the following expression:

$$
E_{m, \text { OOK }}=1.425 \exp \left(-1.28\left(\frac{T}{T_{C}}\right)^{2}\right),
$$

where the symbol period is denoted as $T$ and the channel $10 \%-90 \%$ rise-time is denoted as $T_{C}$ [5]. Methods of calculation of the rise-time for a given system are described in [5]. Under the same assumptions about the shape of the pulse and the impulse responses, the worst case eye closure approximation for 4-PAM is given by

$$
E_{m, 4 P A M}=2.85 \exp \left(-1.28\left(\frac{T}{T_{C}}\right)^{2}\right),
$$

which is twice as large as for OOK at the same symbol rate [4]. This approximation comes from the middle eye of 4-PAM eye diagram and does not account for the fact that the top and bottom eye closures are asymmetric, thus in reality the penalties should be slightly higher.

\section{Experiment and discussion}

The investigated system was a back-to-back (BTB) test setup comprising a directly modulated VCSEL operating $850 \mathrm{~nm}$ with $16 \mathrm{GHz}$ modulation bandwidth, a multimode fiber (MMF) patchcord and a photoreceiver integrated with transimpedance amplifier, with $12 \mathrm{GHz}$ modulation bandwidth. The magnitude of the frequency response of this set-up is presented in Fig. 1a. The $-3 \mathrm{~dB}$ bandwidth of the test set-up was around $8.5 \mathrm{GHz}$ and the $-6 \mathrm{~dB}$ bandwidth was around $12 \mathrm{GHz}$. The roll-off of the frequency response was around $15 \mathrm{~dB} /$ decade. The bandwidth of the system was limited by the slowest component (in this case the photoreceiver), according to the well known formula $B W_{\text {system }}^{-2}=B W_{1}^{-2}+B W_{2}^{-2}+B W_{3}^{-2}+\cdots$, which is valid for Gaussian responses. The rise time was calculated to be 49 ps. The theoretical Gaussian response was plotted along with the measured one in Fig. 1a and it turned out to match well, with exception of the high frequency part above $16 \mathrm{GHz}$, where the roll-off of the measured response was faster.

The given system parameters were used to calculate the theoretical sensitivity and ISI penalties for OOK and 4PAM, using the equations presented in Section 2. The sensitivities at $B E R=10^{-9}$ are plotted in Fig. 1b. For the tested system the sensitivity of 4-PAM started to match the sensitivity of OOK (including the ISI penalties) at bit rates above $30 \mathrm{Gbps}$. At these bit rates, 4-PAM also began to experience ISI penalties.

The 4-PAM signal was generated and the BER measured in real time using methods demonstrated in [3]. Extra care was taken to ensure the same amplitude of the electrical signal driving the VCSEL for both OOK and 4-PAM. The BER results for OOK are presented in Fig. $2 \mathrm{a}$ and the BER results for 4-PAM are presented in Fig. 2b. The sensitivities at $\mathrm{BER}=10^{-9}$ are compared in Fig. $1 \mathrm{~b}$. The experimental results agree reasonably well with the theoretical predictions, 


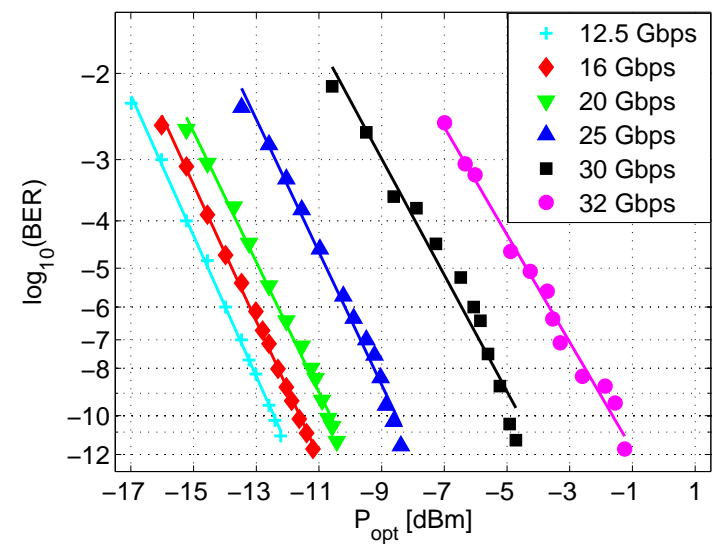

(a)

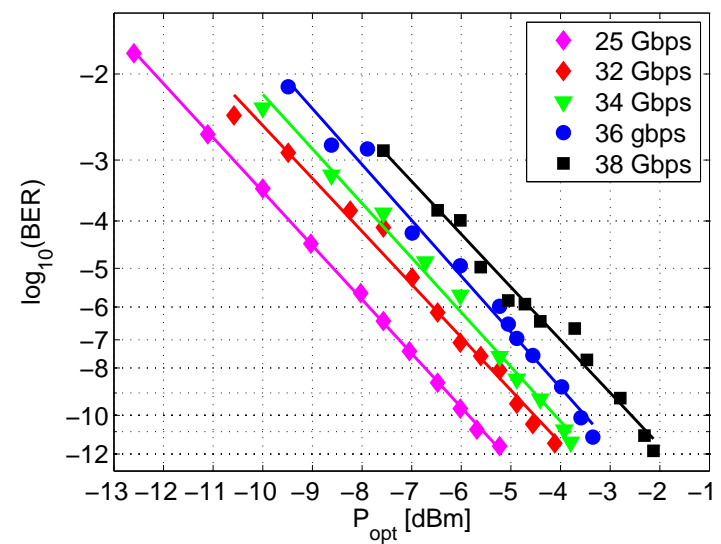

(b)

Fig. 2: Bit error rates measured for OOK (a) and 4-PAM (b).

in the sense that 4-PAM had better sensitivity beyond $30 \mathrm{Gbps}$. In the case of OOK the ISI penalties were worse than theoretical at high bit rates, probably because the signal was affected more by the faster than theoretical roll-off of the magnitude of the frequency response. It can be seen in Fig. 1a that the roll-off increases with frequency. There were also implementation penalties in case of 4-PAM at $25 \mathrm{Gbps}$, which shifted the entire sensitivity curve upwards.

Both OOK and 4-PAM could be used well beyond the $-3 \mathrm{~dB}$ bandwidth of the system, due to the relatively slow roll-off of the frequency response, but as more MMF would be added, the bandwidth would decrease and the roll-off would increase. This would translate into longer rise time. The ISI penalty is dependent on the ratio of the symbol period to the rise time, and thus for longer rise times, the horizontal axis of Fig. 1b would be rescaled to lower bit rates, but the overall shape should remain similar. Therefore, it is expected that with increasing MMF length, 4-PAM would still achieve higher maximum bit rates than OOK.

\section{Conclusions}

In the case of the particular studied system, 4-PAM achieved a higher maximum throughput than OOK, in agreement with theoretical predictions. We obtained bit rates as high as $38 \mathrm{Gbps}$, which to our knowledge is the highest for 4PAM in $850 \mathrm{~nm}$ systems with BER measured down to $10^{-12}$. Maximum achievable bit rate with OOK was 32 Gbps. The absolute ISI penalties were however slightly higher that predicted by the simple theoretical model, which assumed Gaussian pulses and impulse responses. The theoretical Gaussian response modelled well the real frequency response, with an exception of the roll-off at very high frequencies.

\section{References}

1. K. Szczerba, B. -E. Olsson, P. Westbergh, A. Rhodin, J. S. Gustavsson, Å. Haglund, M. Karlsson, A. Larsson, and P. A. Andrekson, "37 Gbps transmission over $200 \mathrm{~m}$ of MMF using single cycle subcarrier modulation and a VCSEL with $20 \mathrm{GHz}$ modulation bandwidth," in Proc. ECOC, Sept. 2010, paper We.7.B.2.

2. S. C. J. Lee, F. Breyer, S. Randel, D. Cardenas, H. P. A. van den Boom, and A. M. J. Koonen, "Discrete multitone modulation for high-speed data transmission over multimode fibers using 850-nm VCSEL," in Proc. OFC, Mar. 2009, paper OWM2.

3. K. Szczerba, P. Westbergh, J. Karout, J. S. Gustavsson, Å. Haglund, M. Karlsson, P. A. Andrekson, E. Agrell, and A. Larsson, "30 Gbps 4-PAM transmission over $200 \mathrm{~m}$ of MMF using an $850 \mathrm{~nm}$ VCSEL," Opt. Express, vol. 19, no. 26, pp. B203-B208, Dec. 2011.

4. K. Szczerba, P. Westbergh, J. Karout, J. S. Gustavsson, Å. Haglund, M Karlsson, P. A. Andrekson, E. Agrell and A. Larsson, "4-PAM for high-speed short-range optical communications," accepted to J. Opt. Commun. Netw., vol. 4, no. 11, Nov. 2012.

5. D. Cunningham, M. Nowell, D. Hanson, L. Kazovsky, "The IEEE 802.3 z Worst Case Link Model for Optical Physical Media Dependent Specification," http://www.ieee802.org/3/z/public/presentations/mar1997/DCwpaper.pdf. 